\title{
Double exponential lightning current model adaptive filter design*
}

\author{
Hua-Fu Li ${ }^{1}$, Wei He ${ }^{1 \dagger}$, Qi-Qi He ${ }^{2}$ and Jin-Ji Zheng ${ }^{1}$ \\ ${ }^{1}$ School of Physics and Electronic Information, Yunnan Normal University, ${ }^{2}$ \\ School of Computer and Information, Yunnan University \\ Kunming, China \\ tE-mail: he99wei@aliyun.com
}

\begin{abstract}
This paper studies the basic structure and principles of adaptive noise filter proposed for the lightning current filtered LMS algorithm signal interference with the traditional difference is fixed filter. Development of adaptive filtering technology and the rapid development of VLSI technology for noise reduction study provides a theoretical and technical support. It is able to detect and extract the useful signal from the noise environment. LMS has the ability to automatically adjust its own parameters and it requires little or no prior knowledge of the undesired signal and noise. At same time, by means of MATLAB that lightning current signal by a double exponential model were to dry process simulation. Verification indicates that the filter has a good performance and this some practical value in the lightning current waveform analysis.
\end{abstract}

Keywords: Adaptive Filter; Lightning Current Waveform; Matlab.

\section{Introduction}

Adaptive control means is a control strategy by changing the behavior of the system to adapt to changes in the process dynamics and environmental conditions. Since the 1950s, it made a first adaptive control system by the Massachusetts Institute of Technology (due to flight control that MIT professor H.P. Whitaker first proposed model reference adaptive control method and use it to solve the problem of driving the vehicle[1]). It has appeared in a variety of different types of adaptive control system such as model reference adaptive control systems, self-tuning control system, optimization control system, variable structure control systems and intelligent self-adaptive control system [2] Adaptive filter and information theory, detection and optimal estimation theory and filter theory is closely related and it is an important branch of signal processing disciplines [3]. With the rapid development of VLSI technology and computer technology and adaptive filter theory itself constantly improve which

\footnotetext{
* This work is supported by National Natural Science programs (51267021) and College students' innovative entrepreneurial training programs (2015).
} 
making its application more and more widely. It has throughout communications, speech signal processing, image processing, pattern recognition, system identification and automatic control and other fields. It is one of the most active areas of research [4]. It is one of the best filtering methods developed on the basis of linear filtering Wiener filter and Kalman filtering [5]. Because of its greater flexibility and better filtering performance so resulting in engineering practice it has been widely used in information processing technology. Object of study is the adaptive filtering systems or processes with uncertain information. Here the "uncertainty" refers to the mathematical model studied the process of information processing and environmental concerns are not fully defined. In the face of these objective existences of a wide range of uncertainties that an integrated approach to the information process and makes a number of specific performance indicators to achieve optimal or near optimal which is to be adaptive filtering problem [6].

In the adaptive noise cancellation system that the adaptive filter does not require prior knowledge of the input signal. It is the use of a time before the input signal has been received is eligible parameters to adjust the current time filter parameters to adapt the signal and noise or unknown statistical properties change with time in order to achieve optimal filtering[7]. Currently adaptive noise cancellation system is widely used such as access to capture automatically track noise sources and excellent performance high signal to noise ratio [8]. Used an adaptive noise cancellation systems for an air combat environment may greatly improve the quality of aeronautical communications. In medicine, for canceling the fetus in the mother's ECG heart sound from the signal applied to the mother's abdomen made reference input which is the fetal heartbeat and the mother's heart sounds superimposed signal obtained from the mother breast plus adaptive filter input output system is the best estimate of the fetal heart sounds[8]. In short, to seek convergence speed, low computational complexity, good numerical stability and practical adaptive filtering algorithm researchers constantly strive for the goal.

\section{Principle and Calculation}

In the random signal processing Wiener filter is frequently used and Kalman filter two filters. Weiner filter which according to all past and current observational data stationary random signal to estimate the current value of the signal. At least mean conditional variance to obtain the transfer function or impulse response, it is an optimal linear filtering method that parameters are fixed for stationary random signals. Kalman filter which is based on the current observation time data of the previous time recursive algorithm for filtering data processing time of the predicted value. It automatically adjusts the shock 
response itself or that automatically adjust the digital filter coefficients to adapt to changes in the characteristics of the signal so as to achieve optimal filtering. It is time-varying parameters, applicable to non-stationary random signals. However, only if the statistical properties of the noise signal is known a priori, the two filters in order to obtain optimal filtering. However, in practice it is often unable to obtain a priori knowledge of these statistical properties or statistical properties are changing with time. Thus, Wiener or Kalman filter cannot achieve optimal filtering. In this case, the adaptive filter to provide superior performance. The so-called adaptive filtering is the use of a time before the filter parameters have been obtained results etc. It is the automatic adjustment of the filter parameters of the current time and the noise signal to accommodate unknown or time-varying statistical properties in order to achieve optimal filtering. Adaptive filter is essentially a can regulate its own transmission characteristics in order to achieve optimal Wiener filter. Adaptive filter does not require a priori knowledge about the input signal, a small amount of calculation, especially for real-time processing.

It is an adaptive filter algorithm for a variety. There are minimum mean square criterion (Least Mean Square, LMS) algorithm and its various modifications algorithm, based on least squares criterion (Recursive Least Square, RLS) algorithm fast transversal filter (fast transverse filter, FTF) algorithm. Although the LMS algorithm and RLS algorithm than the FTF algorithm convergence slow but considering, LMS algorithm and RLS compared FTF algorithm is simple and small calculation (LMS computation $\propto M$, computational RLS $\propto M^{2}$, FTF computation amount $\propto(7 \sim 10) M$, where $\mathrm{M}$ is a filter weighting coefficient number). So the LMS is easy to implement.

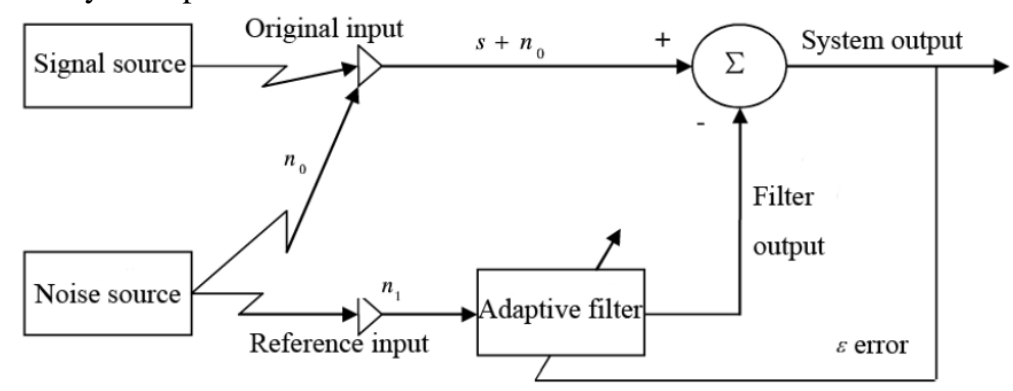

Fig. 1. A block diagram of an adaptive filter

As shown in Fig.1, the signal transmitted from the channel of the signal received by the sensor. In addition to the received signal that the sensor will also receive an unrelated noise $n_{0}$ which superimposed on the signal $s+n_{0}$ to consist canceller Original input. The second sensors, signal reception and irrelevant but 
in some way associated with unknown noise $n_{0}$ and $n_{1}$. This sensor is provided to offset the Reference input, the noise $n_{1}$ be filtered to produce approximately $n_{0}$ replica $y$ will the output from the original input $s+n_{0}$ subtracted to produce the system output.

\section{Double Exponential Model Lightning Current Filter Simulation}

Adaptive filter has a good working environment under unknown statistics and the ability to track the input changes over time. Although for different applications have different implementation structures but they all had one basic feature: input vector $x(n)$ and the desired response $d(n)$ are used to calculate the estimated error $e(n)$, namely:

$$
e(n)=d(n)-x(n)
$$

Using this error signal construct an adaptive algorithm performance function (such as the mean square error MSE) and the performance of this function adaptively updated continuously with the input data, the goal is to minimize this cost function, in the process constantly updated adjustment filter parameters of the filter, so that the optimal parameter in the previous guidelines to minimize the performance function used to achieve filtering effect, adaptive process.

Using MATLAB programming that the lightning current through the double exponential model of random noise is added. After the adaptive filter, a filtered output signal. Wherein the double exponential model:

$$
i(t)=I_{m} k\left(e^{-\alpha t}-e^{-\beta t}\right)
$$

Among them, $\alpha=1.624 \times 10^{4}, \beta=0.456 \times 10^{6}, k=1.05$.Get the simulation shown in Fig.2, the error signal is shown in Fig.3.
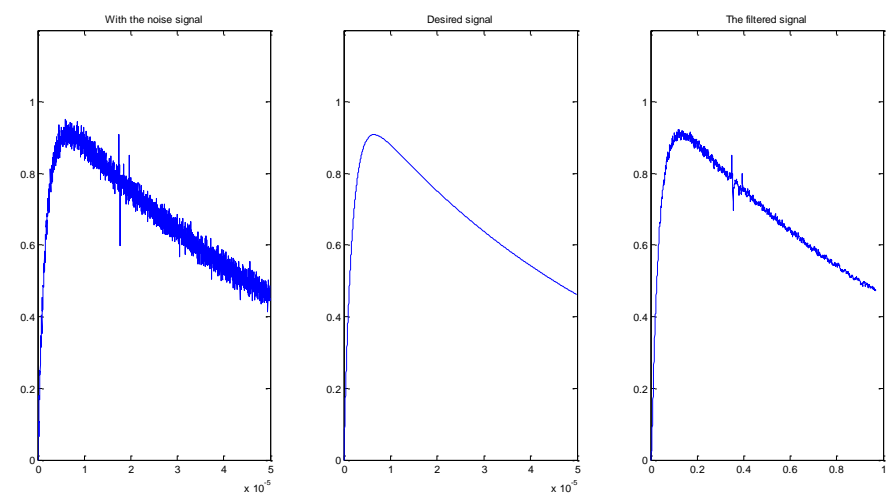

Fig. 2. Double exponential lightning current model adaptive filter 


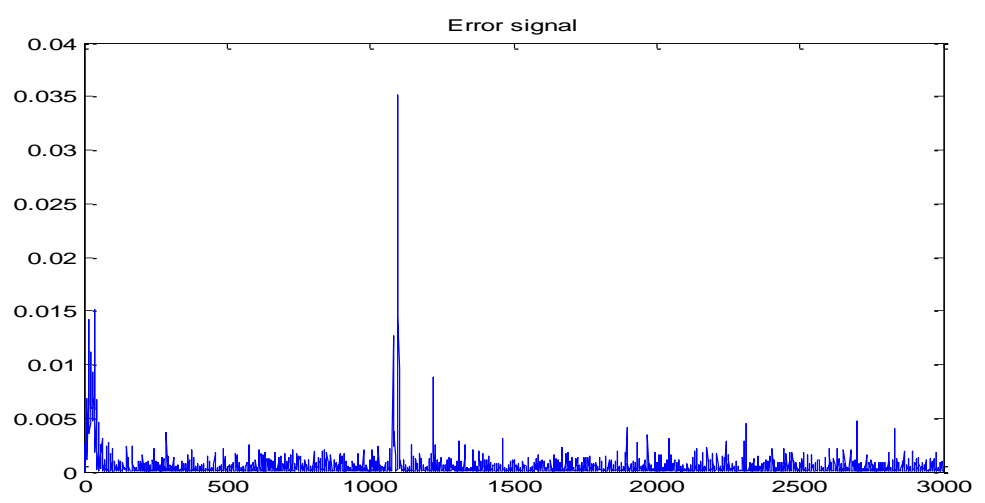

Fig. 3. Error signal

The above figure can be seen through the noise signal of the adaptive filter can well be suppressed. The useful signal separation obviously is a solution scheme lightning current signal filtering. Today is an important foundation for adaptive filtering signal processing. Fast pace of development in recent years, he made a wide range of applications in various fields mainly in five areas: adaptive filtering and inverse filtering; system identification; adaptive equalization: adaptive echo cancellation; communication noise elimination. In practical problems, the urgent need for effective research and practical adaptive filtering algorithm. This paper studied the basic structure and principles of adaptive noise filter proposed for the lightning current filter out the interference signal LMS algorithm, using MATLAB simulation through the lightning current signal double exponential models were treated to dry. To prove that the filter has a good performance, the lightning current waveform analysis has some practical value.

\section{References}

1. Gao Ying. An adaptive filtering algorithm based on least squares criterion [J].Guangzhou University, 2007, 15 (2): 32-34.

2. Ginger up, Tu Qingping. Adaptive noise cancellation applied research and simulation technology [J].Computer Simulation, 2008, (2):311-314.

3. Bernard Widrow, Samuel DStearns with, Wang Yongde, Long Hui Xian. Adaptive signal processing [M].Beijing: Mechanical Industry Press, 2008.

4. Liu Chunjie. Real-Time Adaptive Noise Design and Implementation of [D].offset by Qingdao Ocean University, 2001. 
5. Luo Pengfei, Yang Shihai, Zhu Guofu Tan.achieve full digital signal processing method of the second edition [M].Beijing: Electronic Industry Press, 2004.

6. PauloS.R.Diniz. adaptive filter algorithm and implementation [M].Beijing: Electronic Industry Press, 2010.

7. Yin Haoqiong.communication system echo cancellation study [D]. Beijing University of Posts and Telecommunications, 2006.

8. Hou Ebel, Liuxiong Fei.high-speed sampling and adaptive filter system simulation [J].Detection \& Control, 2007, No.12106: 24-27. 